\title{
Effect of Visual Acuity in Different Amount of Deviation of Exotropia
}

\author{
Partha $\mathrm{HC}^{\mathbf{1}^{*}}$, Brinda $\mathrm{HS}^{2}$ \\ 1M.Optom, Department of Optometry, Shree Satchandi Jankalyan Samiti Netra Prasikshan Sansthan, Pauri, Affliated to \\ Uttarakhand State Medical Faculty, Dehradun, India \\ ${ }^{2}$ B.Optom, Department of Optometry, Gujarat University, Gujarat, India
}

Received: 14 March, 2018; Accepted: 25 April, 2018; Published: 03 May, 2018

*Corresponding author: Partha HC, M.Optom, Department of Optometry, Shree Satchandi Jankalyan Samiti Netra Prasikshan Sansthan, Pauri, Affliated to Uttarakhand State Medical Faculty, Dehradun, India, E-mail: optometrypublish@gmail.com

\begin{abstract}
Purpose: The aim of the present study is to compare the effect of visual acuity in different amount of deviations in Exotropia.

Methods: A pilot, cross sectional, observational study was performed at tertiary eye care centers. Subjects with Ocular deviation between 10 to 40 prism diopters, Corrected distance Visual Acuity should be greater than 6/18 and Age should be between 10 to 40 years of age were included in the study. Visual Acuity was assessed with Log Mar chart.

Results: 30 subjects were included in the study. Out of that, 16 subjects were in the age group of 11-20 years, 12 subjects were in the age group of 21-30 years and 2 subjects were in the age group of $31-40$ years. $60 \%$ subjects were Female and $40 \%$ subjects were Male. The mean visual acuity was considered in each amount of deviation. It shows that visual acuity will be deteriorated more as ocular deviation increases in cases of Exotropia.
\end{abstract}

Conclusions: In ocular deviation of Exotropia, as amount of deviation increases, visual acuity decreases gradually.

Keywords: Exotropia; Amount of Deviation; Visual Acuity;

\section{Introduction}

In case of Exodeviation images of an object fall on the Para foveal region and deteriorate the visual performance. Esodeviation is more harmful as compared to Exodeviation, because intermittent timings are very less in Esodeviation as compared to Exodeviation. Because in the foveal region the cone cell density is much more compare to para foveal region. That's why in case of Exodeviation the images of an object is shifted to the temporal para foveal region and this image shifted is completely depends on the ocular deviation. In case of high ocular deviation the image shift will be high. Image will shift towards the temporal region and due to decrease in cone cell number, visual performance is deteriorated. Due to less intermittent stages in Exodeviation compare to Esodeviation there may be less chance to become Amblyopia in Exodeviation. That's why there may be strong correlation with the ocular deviation with Visual Acuity due to anatomical anomalies of the photo receptor cells.

\section{Methodology}

A pilot, cross sectional, observational study was performed at tertiary eye care centers. Subjects with Ocular deviation between 10 to 40 prism diopters, Corrected distance Visual Acuity should be greater than 6/18 and Age should be between 10 to 40 years of age were included in the study. Individuals with any other systemic disease (specially which can affect study), Individuals with any other Ocular Pathology, with any active ocular infection, any ocular anomalies like Corneal Scar etc ,ocular deviation if less than 10 degree and Significant amount of amblyopic patient were excluded from the study. Full refractive correction along with detailed fund us evaluation was performed in each and every patient. Visual acuity was assessed with Log Mar chart in different amounts of deviation in Exotropia. Data was analyzed using SPSS software version 20.

\section{Results}

30 subjects were included in the study. (Graph 1) shows distribution of subjects in various age groups.16 subjects were in the age group of 11-20 years, 12 subjects were in the age group of 21-30 years and 2 subjects were in the age group of 31-40 years. (Graph 2) shows gender wise distribution of the subjects. $60 \%$ subjects were Female and $40 \%$ subjects were Male. (Graph 3 ) shows comparison of Visual Acuity for different amount of

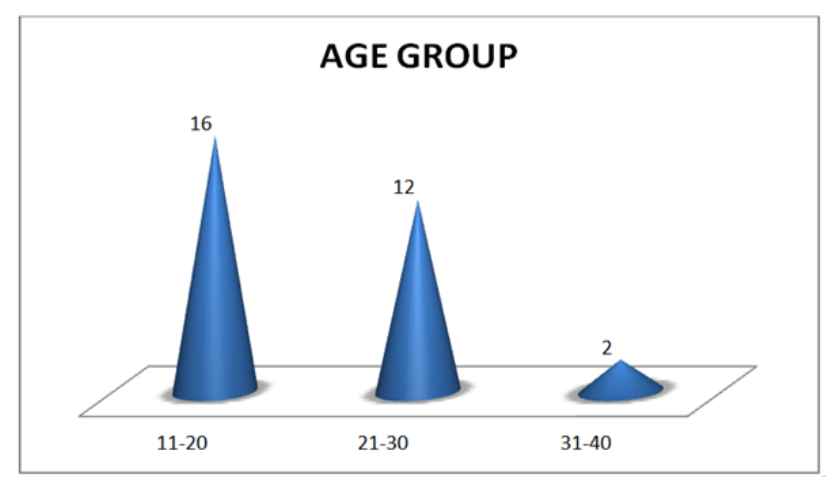

Graph 1: Shows Age Wise Distribution of the Subject 


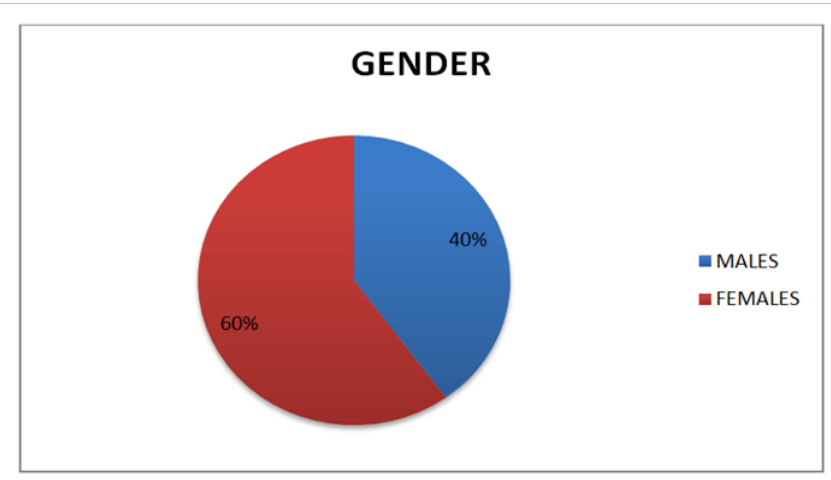

Graph 2: Shows Gender Wise Distribution of the Subjects

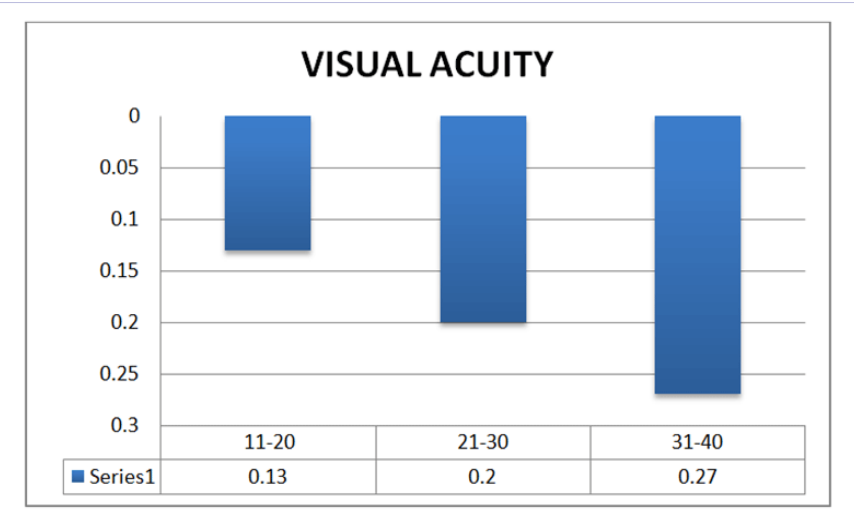

Graph 3: Shows comparison of Visual Acuity for different amount of deviation for Exotropia

deviation for Exotropia. The mean visual acuity was considered in each amount of deviation. It shows that visual acuity will be deteriorated more as ocular deviation increases in cases of Exotropia.

\section{Discussion}

Visual acuity of a normal person is $0.00 \log$ units. In this study, according to statistical analysis it shows that visual acuity was deteriorated to $0.13 \log$ units in the ocular deviaition of 11-20 prism diopters, 0.2 log units in 21-30 prism diopters and 0.27 log units in 31-40 prism diopters. It shows that as there in increase in the amount of ocular deviation, visual acuity with decrease gradually. In case of ocular deviation images of an object are falling on para foveal region and due to inequality of the number of the cone cells in the macular region there may be chances of the vision deterioration with increasing ocular deviation. In case of Exodeviation the images of an object is placed at the temporal retinal side and due to its intermittent stages is very high compare to Esodeviation, there have less chances to become Amblyopia. Thus statistically it has been proved that with increasing ocular deviation visual Acuity has been deteriorated proportionately but compare to Esodeviation with same amount of deviation Visual Acuity is more deteriorated in Esodeviation compare to exodeviation.

\section{Conclusion}

In ocular deviation of Exotropia, as amount of deviation increases, visual acuity decreases gradually.

\section{Consent}

Oral/ written consent was obtained from patient as well as from tertiary eye care centers.

\section{Ethical Approval}

It is not applicable.

\section{Competing Interests}

Authors have declared that no competing interests exist.

\section{References}

1. Wright KW, Spiegel PH, Thompson L. Handbook of Pediatric Strabismus and Amblyopia. First Ed, 2006.

2. Hui Zhu, Jia-Jia Yu, Rong-Bin Yu, Hui Ding, Jing Bai, Ji Chen, et al. Association between Childhood Strabismus and Refractive Error in Chinese Preschool Children. PLoS One. 2015;10(3):e0120720.

3. Rajavi Z, Sabbaghi H, Baghini AS, Yaseri M, Sheibani K, Norouzi G. Prevalence of Color Vision Deficiency and its Correlation with Amblyopia and Refractive Errors among Primary School Children. J Ophthalmic Vis Res. 2015;10(2):130-138.

4. Tandon AK, Velez FG, Isenberg SJ, Demer JL, Pineles SL. Binocular Inhibition in Strabismic Patients is Associated with Diminished Quality of Life. J AAPOS. 2014;18(5):423-426.

5. Ye XC, Pegado V, Patel MS, Wasserman WW. Strabismus genetics across a spectrum of eye misalignment disorders. Clin Genet. 2014;86(2):103-111.

6. Koçak-Altintas AG, Satana B, Koçak I, Duman S. Visual Acuity and Color Vision deficiency in Amblyopia. Eur J Ophthalmol. 2000;10(1):77-81.

7. Freeman AW, Nguyen VA, Jolly N. Components of Visual Acuity Loss in Strabismus. Vision Res. 1996;36(5):765-774. 